Homology, Homotopy and Applications, vol.4(1), 2002, pp.29-42

\title{
SOME RESULTS ON CENTRAL EXTENSIONS OF CROSSED MODULES
}

\author{
A. M. VIEITES AND J. M. CASAS \\ (communicated by Hvedri Inassaridze)
}

\begin{abstract}
In this paper certain central extensions of crossed modules are classified. For these extensions, we obtain several results which extend the classical ones for central extensions of groups. In particular, central stem extensions of a perfect crossed module are classified in terms of a second integral homology crossed module.
\end{abstract}

\section{Introduction}

Several homology and cohomology definitions have been given during the last years: Ellis [5] and Baues [1] define the (co)homology of a crossed module $(M, P, \mu)$ with coefficients in a $P / \mu(M)$-module $A$ as the cohomology of its classifying space. Ladra and Grandjeán [11] define, for a crossed module, the two first crossed modules of homology; associated to an extension of crossed modules, they obtain a five-term exact sequence connecting $H_{1}$ and $H_{2}$. For a crossed module, they also get a generalized Hopf formula and in [7], for a prefect and aspherical crossed module $(T, G, \partial)$, they find a universal central extension whose kernel is the invariant $\mathrm{H}_{2}(T, G, \partial)$. In [16] a generalization to crossed modules of the Eilenberg-MacLane cohomology groups through extensions is given.

For a given crossed module $(M, P, \mu)$, we introduce in [18] cohomological $\delta$ functors $\operatorname{Opext}^{n}((M, P, \mu),-), n=1,2$, from $(M, P, \mu)$-modules to abelian groups which generalize the functors of $n$-fold extensions of groups $\operatorname{Opext}^{n}(G,-)$ [10]. Moreover, we obtain an exact and natural eight term exact sequence related to an extension of crossed modules which gives rise to an eight term exact sequence in group cohomology [14], as a particular case.

We begin section 2 by summarizing several results from [4]: we start by recalling that the category of crossed modules $\mathcal{C M}$ is tripleable over the category of sets, so that it is an algebraic category; this result involves new concepts of free and projective crossed modules and leads to a cotriple (co)homology theory for crossed modules; we recall from $[\mathbf{1 1}]$ and $[\mathbf{1 6}, \mathbf{1 8}]$ the five-term exact sequences for homology with trivial coefficients and for cohomology, respectively. Also from [4], we consider, in section 3, the classification of central extensions of a crossed module

Supported by Government of Galicia (GRANT: PGIDT00PXI 37101PR).

Received September 21, 2002, revised April 10, 2002; published on May 2, 2002.

2000 Mathematics Subject Classification: 18G50, 18G99, 20J10.

Key words and phrases: crossed module, central extension, stem extension.

(C) 2002, A. M. Vieites and J. M. Casas. Permission to copy for private use granted. 
by its second cohomology group, and an universal coefficient theorem for crossed modules cohomology, which extends the classical one for cohomology of groups [9]. Finally, in the last section, we study two of these kinds of central extensions: stem extensions and stem covers and we prove several results for central extensions of perfect crossed modules, by generalizing the classical ones for central extensions of groups, being our main result Theorem 4 where it is shown that stem extensions of a perfect crossed module $(M, P, \mu)$ are in bijective correspondence with the subcrossed modules of an appropriately defined $\mathrm{H}_{2}(M, P, \mu)$ and that the lattice of subcrossed modules of $\mathrm{H}_{2}(M, P, \mu)$ is equivalent to the category of stem extensions of $(M, P, \mu)$.

\section{Preliminaries}

For the faithful functor to groups, $\mathcal{V}: \mathcal{C M} \rightarrow \mathcal{G},(T, G, \partial) \rightarrow T \times G$, a left adjoint is given in [4] as follows: if $H$ is any group, get the free product group $H * H$ with the injections

$$
u_{i}: H \rightarrow H * H, \quad i=1,2
$$

and let $\bar{H}=\operatorname{Ker}\left(p_{2}: H * H \rightarrow H\right)$ be the kernel of the retraction $p_{2}$, determinated by the conditions $p_{2} u_{1}=0$ and $p_{2} u_{2}=i d_{H}$. The triple $(\bar{H}, H * H, i n)$ is a crossed module with the inclusion as boundary map. The functor $H \rightarrow(\bar{H}, H * H, i n)$ is left adjoint to $\mathcal{V}$.

With the usual forgetful functor $\mathcal{G} \rightarrow \mathcal{S}$ et, there is the underlying set functor $\mathcal{U}: \mathcal{C M} \rightarrow \mathcal{S}$ et,$\quad(T, G, \partial) \rightarrow T \times G$. Since the forgetful functor $\mathcal{G} \rightarrow \mathcal{S e t}$ has the free group functor, $X \rightarrow F(X)$, as a left adjoint, the functor $\mathcal{U}: \mathcal{C M} \rightarrow \mathcal{S}$ et has a left adjoint $\mathcal{F}: \mathcal{S} e t \rightarrow \mathcal{C M}$ given by $X \rightarrow \mathcal{F}(X)=(\overline{F(X)}, F(X) * F(X)$, in $)$, [4].

In that paper it is also shown that $\mathcal{U}$ is tripleable and it follows from this fact that, in the category of crossed modules, regular epimorphisms are just those homomorphisms $\left(f_{1}, f_{2}\right):(T, G, \partial) \longrightarrow\left(T^{\prime}, G^{\prime}, \partial^{\prime}\right)$ such that both $f_{1}$ and $f_{2}$ are onto maps. Hence, for any set $X$, the free crossed module on $X, \mathcal{F}(X)$, is projective, and every crossed module $(T, G, \partial)$ admits a projective representation by means of the free crossed module on its underlying set and the counit of the adjunction. From [4] it is known that the category of abelian crossed modules, $\mathcal{C M}_{a b}$, is equivalent to the category of right modules over the ring of matrices

$$
\left(\begin{array}{ll}
\mathbb{Z} & 0 \\
\mathbb{Z} & \mathbb{Z}
\end{array}\right)=\left\{\left(\begin{array}{ll}
a & 0 \\
b & c
\end{array}\right) ; a, b, c \in \mathbb{Z}\right\}
$$

and it has global dimension equal to 2 . This result implies, in particular, that $\mathcal{C M}_{a b}$ has enough projective and injective objects.

We consider now the abelianization functor $\mathcal{A} b: \mathcal{C M} \rightarrow \mathcal{C M}_{a b}$, that to each crossed module $\mathbf{T}=(T, G, \partial)$ associates its abelianization, that is, $\mathbf{T}_{a b}=\mathbf{T} /[\mathbf{T}, \mathbf{T}]=$ $(T /[G, T], G /[G, G], \bar{\partial}),[\mathbf{1 3}]$, and to each morphism the induced one. This functor $\mathcal{A} b$ is left adjoint to the inclusion functor $\mathcal{U}: \mathcal{C M}_{a b} \rightarrow \mathcal{C M}$. This follows from the universal property [13] of the commutator crossed submodule. Therefore, $\mathcal{A} b$ preserves surjective morphisms, as they are the conormal maps of $\mathcal{C M}$. In [11] is 
defined the first homology crossed module of a crossed module $\mathbf{T}$ as $\mathrm{H}_{1}(\mathbf{T})=\mathbf{T}_{a b}$. Examples:

1. If $N$ is a normal subgroup of $G, \mathrm{H}_{1}(N, G, i)=\left(N /[G, N], \mathrm{H}_{1}(G), \bar{i}\right)$.

2. $\mathrm{H}_{1}(G, G, i d)=\left(\mathrm{H}_{1}(G), \mathrm{H}_{1}(G), i d\right)$ and $\mathrm{H}_{1}(1, G, i)=\left(1, \mathrm{H}_{1}(G), i\right)$.

3. If $A$ is a $G$-module, then $\mathrm{H}_{1}(A, G, 0)=\left(\mathrm{H}_{0}(G, A), \mathrm{H}_{1}(G), 0\right)$.

The second homology crossed module of a crossed module is also introduced in [11] by using a particular kind of presentations called $\varepsilon$-projective. They show that, with their definition, this second homology crossed module is indepent of the chosen presentation. If we consider a projective presentation using the projective crossed modules which are introduced in [4], we get the results of [11] using analogous proofs; it is enough to consider projective presentations instead of $\varepsilon$-projective presentations. Essentially, given a projective presentation $\mathbf{B}+\longrightarrow \mathbf{U} \longrightarrow \mathbf{1} \rightarrow \mathbf{T}$ of the crossed module $\mathbf{T}$, the second homology crossed module of $\mathbf{T}$ is defined as $\mathrm{H}_{2}(\mathbf{T})=\frac{\mathbf{B} \cap[\mathbf{U}, \mathbf{U}]}{[\mathbf{U}, \mathbf{B}]}$, where $[\mathbf{U}, \mathbf{B}]=([E, U][V, B],[V, E], \omega)$, if $\mathbf{U}=(U, V, \omega)$ and $\mathbf{B}=(B, E, \zeta)$. This definition is independent, up to isomorphism, of the chosen projective presentation and the correspondence $\mathbf{T} \rightarrow \mathrm{H}_{2}(\mathbf{T})$ defines a functor $\mathrm{H}_{2}$ : $\mathcal{C M} \rightarrow \mathcal{C M}_{a b}$. Moreover, if $\mathbf{T}$ is a projective crossed module, then $\mathrm{H}_{2}(\mathbf{T})=\mathbf{1}$.

Examples: $\mathrm{H}_{2}(G, G, i d)=\left(\mathrm{H}_{2}(G), \mathrm{H}_{2}(G), i d\right), \mathrm{H}_{2}(1, G, i)=\left(1, \mathrm{H}_{2}(G), i\right)$.

An extension of $\mathbf{N}=(N, R, \nu)$ by $\mathbf{M}=(M, P, \mu)$ is an exact sequence of crossed

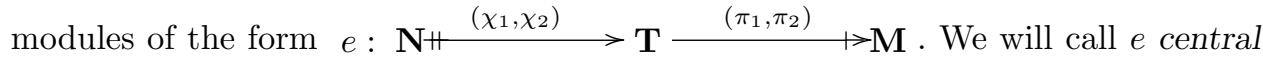
if $\operatorname{Im}\left(\chi_{1}, \chi_{2}\right) \subseteq \mathrm{Z}(\mathbf{T})=\left(T^{G}, \mathrm{Z}(G) \cap \operatorname{st}_{G}(T), \partial\right)$.

When $\mathbf{N}$ is abelian any extension $e$ induces an action of $\mathbf{M}$ on $\mathbf{N},[\mathbf{1 3}]$.

For a $\mathbf{M}$-module $\mathbf{N}$ (that means $\mathbf{N}$ is an abelian crossed module such that $\mathbf{M}$ acts on it) the following definition can be given: an extension $e$ of $\mathbf{N}$ by $\mathbf{M}$ is called a $\mathbf{M}$-extension if the induced action is the given action of $\mathbf{M}$ on $\mathbf{N}$. In this case it can be shown that $e$ is central if and only if the induced action is the trivial one.

An extension morphism is a commutative diagram in $\mathcal{C M}$ of the form

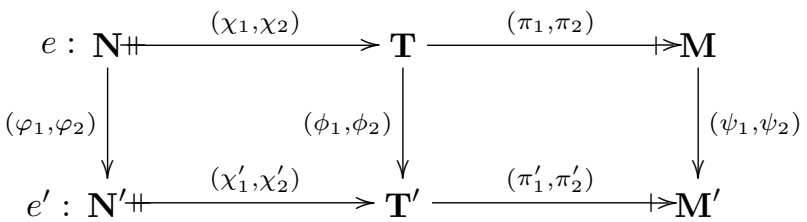

We will denote such morphisms as $\left(\left(\varphi_{1}, \varphi_{2}\right),\left(\phi_{1}, \phi_{2}\right),\left(\psi_{1}, \psi_{2}\right)\right): e \rightarrow e^{\prime}$, and we will say that two extensions, $e$ and $e^{\prime}$, of $\mathbf{N}$ by $\mathbf{M}$ are congruent if there exists an extension morphism $\left(i d_{\mathbf{N}},-, i d_{\mathbf{M}}\right): e \rightarrow e^{\prime}$. This is an equivalence relation on the set of $\mathbf{M}$-extensions. Let Opext $(\mathbf{M}, \mathbf{N})$ be the set of equivalence classes. When we consider a trivial $\mathbf{M}$-module $\mathbf{N}$, the $\mathbf{M}$-extensions are central extensions; in this case, $\operatorname{Cext}(\mathbf{M}, \mathbf{N})$ denotes the set $\operatorname{Opext}(\mathbf{M}, \mathbf{N})$.

Theorem 1. [7] Let $e: \mathbf{N} \mathbb{H}^{\left(\chi_{1}, \chi_{2}\right)} \longrightarrow \mathbf{T} \stackrel{\left(\pi_{1}, \pi_{2}\right)}{\longrightarrow} \longrightarrow \mathbf{M}$ be an extension, then 
there exists the following natural exact sequence

$$
\begin{gathered}
\mathrm{H}_{2}(\mathbf{T}) \longrightarrow \mathrm{H}_{2}(\mathbf{M}) \stackrel{\theta_{\star}(e)}{\longrightarrow}(N /[G, N][R, T], R /[G, R], \bar{\nu}) \\
\longrightarrow \mathrm{H}_{1}(\mathbf{T}) \stackrel{\left(\pi_{1}, \pi_{2}\right)_{\star}}{\longrightarrow} \mathrm{H}_{1}(\mathbf{M}) \longrightarrow \mathbf{0}
\end{gathered}
$$

When $e$ is central, $\mathbf{N}$ appears instead of $\mathbf{N} /[\mathbf{N}, \mathbf{T}]$ in the previous exact sequence. Example: Whatever way we regard a group $G$ as a crossed module either $(1, G . i)$ or $(G, G, i d)$ we get the five-term exact sequence in integral homology of groups [9]

$$
\mathrm{H}_{2}(G) \longrightarrow \mathrm{H}_{2}(P) \longrightarrow \mathrm{H}_{1}(G) \longrightarrow \mathrm{H}_{1}(P) \longrightarrow 0 .
$$

From Theorem 1, and by considering a projective presentation of $\mathbf{T}$ of the form $\mathbf{B}+\longrightarrow \mathbf{U} \longrightarrow\left(h_{1}, h_{2}\right) \quad \gg \mathbf{T}$, it is shown that $\operatorname{Ker}\left(\pi_{1}, \pi_{2}\right)_{\star}=\frac{\mathbf{B}}{[\mathbf{U}, \mathbf{B}]}$ in $[\mathbf{1 1}]$.

Theorem 2. [17] Let $e: \mathbf{N} \mathbb{H}^{\left(\chi_{1}, \chi_{2}\right)} \longrightarrow \mathbf{T} \stackrel{\left(\pi_{1}, \pi_{2}\right)}{\longrightarrow} \longrightarrow \mathbf{M}$ be a $\mathbf{M}$-extension, then there exists the following natural exact sequences of abelian groups

i) $0 \longrightarrow \operatorname{Der}(\mathbf{M}, \mathbf{N}) \longrightarrow \operatorname{Der}(\mathbf{T}, \mathbf{N}) \longrightarrow \operatorname{Hom}_{\mathbf{M}}(\mathbf{N}, \mathbf{N})$

$\stackrel{\theta^{\star}(e)}{\longrightarrow} \operatorname{Opext}(\mathbf{M}, \mathbf{N}) \longrightarrow \operatorname{Opext}(\mathbf{T}, \mathbf{N})$,

ii) $0 \longrightarrow \frac{\operatorname{Der}(\mathbf{M}, \mathbf{N})}{\operatorname{IDer}(\mathbf{M}, \mathbf{N})} \longrightarrow \frac{\operatorname{Der}(\mathbf{T}, \mathbf{N})}{\operatorname{IDer}(\mathbf{T}, \mathbf{N})} \longrightarrow \operatorname{Hom}_{\mathbf{M}}(\mathbf{N}, \mathbf{N})$

$\stackrel{\theta^{\star}(e)}{\longrightarrow} \operatorname{Opext}(\mathbf{M}, \mathbf{N}) \longrightarrow \operatorname{Opext}(\mathbf{T}, \mathbf{N})$.

This theorem gives us similar exact sequences that in group category, [2, 9].

\section{Several kinds of central extensions}

Now we define and classify certain central extension of crossed modules $e$ in terms of the morphism $\theta_{\star}(e)$ in Theorem 1:

Definition 1. The central extension e is called a commutator extension if $\theta_{\star}(e)$ is the zero map.

It follows from Theorem 1 that $\theta_{\star}(e)=0$ if and only if the following sequence is an exact sequence $\mathbf{N}+1 \rightarrow \mathbf{M}_{a b}$.

Theorem 3. The following statements are equivalent:

i) $\theta_{\star}(e)=0$,

ii) $\left(\pi_{1}, \pi_{2}\right)_{\star}:[\mathbf{T}, \mathbf{T}] \rightarrow[\mathbf{M}, \mathbf{M}]$ is an isomorphism,

iii) $\mathbf{N} \cap[\mathbf{T}, \mathbf{T}]=\mathbf{0}$. 


\section{Proof:}

It is enough to consider the following commutative diagram given by the cross lemma [16]

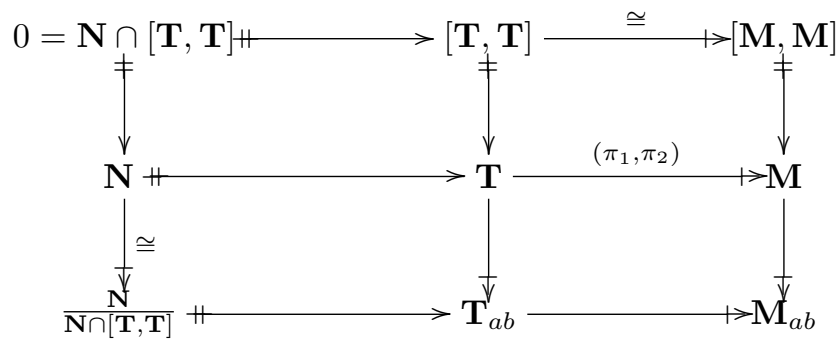

and to keep in mind that $\left(\pi_{1}, \pi_{2}\right)$ induces a surjective map $\left(\pi_{1}, \pi_{2}\right)_{\star}:[\mathbf{T}, \mathbf{T}] \rightarrow$ $[\mathbf{M}, \mathbf{M}]$.

Given $e: \mathbf{N} \# \stackrel{\left(\chi_{1}, \chi_{2}\right)}{\longrightarrow} \mathbf{T} \stackrel{\left(\pi_{1}, \pi_{2}\right)}{\longrightarrow} \longrightarrow \mathbf{M}$ and $\left(\psi_{1}, \psi_{2}\right): \mathbf{N}_{a b} \rightarrow \mathbf{A}=(A, K, \sigma)$ a $\mathbf{M}$-module morphism, the forward induced extension ${ }^{\left(\psi_{1}, \psi_{2}\right)} \mathrm{ab}(e)$ is the extension of $\mathbf{A}$ by $\mathbf{M}$ in the following diagram

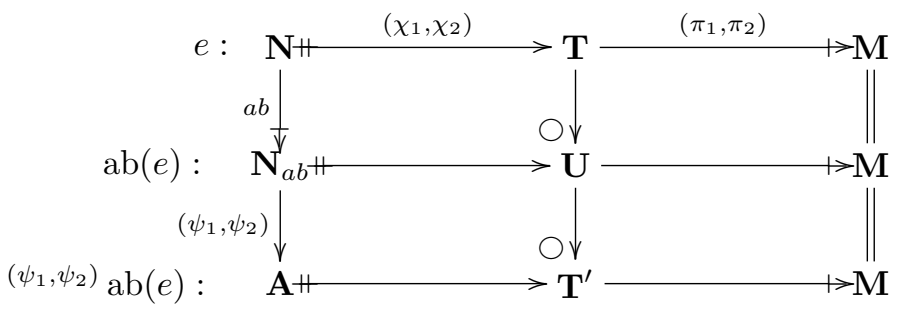

(where $\bigcirc$ denotes de quasi-cocartesian square in $\mathcal{C M}$, [16]). Thus we have an extension morphism $\left(\left(\psi_{1}, \psi_{2}\right) a b,-, i d_{\mathbf{M}}\right): e \rightarrow^{\left(\psi_{1}, \psi_{2}\right)} \mathrm{ab}(e)$. Since $\theta_{\star}$ is natural, we get the following commutative diagram

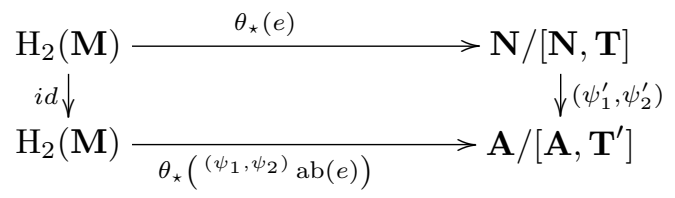

Knowing that $\theta^{\star}: \operatorname{Hom}_{\mathbf{M}}\left(\mathbf{N}_{a b}, \mathbf{A}\right) \rightarrow \operatorname{Opext}(\mathbf{M}, \mathbf{A})$ is given by $\theta^{\star}\left(\psi_{1}, \psi_{2}\right)=$ $\left[\left(\psi_{1}, \psi_{2}\right) \mathrm{ab}(e)\right]$, then $\theta^{\star}\left(\theta_{\star}\left(\psi_{1}, \psi_{2}\right)\right)=\left(\psi_{1}^{\prime}, \psi_{2}^{\prime}\right) \theta_{\star}(e),[\mathbf{1 6}]$.

Theorem 4 (Universal coefficient theorem). [4] For a given trivial M-module A it is shown that:

i) there is a natural exact sequence

$$
\begin{gathered}
0 \longrightarrow \operatorname{Ext}_{\mathcal{C} \mathcal{M}_{a b}}^{1}\left(\mathrm{H}_{1}(\mathbf{M}), \mathbf{A}\right) \stackrel{\phi}{\longrightarrow} \mathrm{H}^{2}(\mathbf{M}, \mathbf{A}) \stackrel{\theta_{\star}}{\longrightarrow} \operatorname{Hom}\left(\mathrm{H}_{2}(\mathbf{M}), \mathbf{A}\right) \\
\longrightarrow \operatorname{Ext}_{\mathcal{C} \mathcal{M}_{a b}}^{2}\left(\mathrm{H}_{1}(\mathbf{M}), \mathbf{A}\right) \longrightarrow \mathrm{H}^{3}(\mathbf{M}, \mathbf{A})
\end{gathered}
$$


ii) if $\mathrm{H}_{2}(\mathbf{M})=\mathbf{0}$, then there is a natural exact sequence

$$
\begin{gathered}
0 \longrightarrow \operatorname{Ext}_{\mathcal{C M}_{a b}}^{2}\left(\mathrm{H}_{1}(\mathbf{M}), \mathbf{A}\right) \longrightarrow \mathrm{H}^{3}(\mathbf{M}, \mathbf{A}) \\
\longrightarrow \operatorname{Hom}\left(\mathrm{H}_{3}(\mathbf{M}), \mathbf{A}\right) \longrightarrow 0
\end{gathered}
$$

iii) if $\mathrm{H}_{i}(\mathbf{M})=\mathbf{0}$, for all $1<i \leqslant n$ and $n \geqslant 2$ then

$$
\begin{gathered}
\mathrm{H}^{i}(\mathbf{M}, \mathbf{A})=\mathbf{0}, \text { for all } 3 \leqslant i \leqslant n, \\
\mathrm{H}^{n+1}(\mathbf{M}, \mathbf{A}) \cong \operatorname{Hom}\left(\mathrm{H}_{n+1}(\mathbf{M}), \mathbf{A}\right) .
\end{gathered}
$$

iv) Let $\mathbf{A} \# \longrightarrow \mathcal{I}^{\bullet}$ be an injective resolution of $\mathbf{A}$ in $\mathcal{C M}_{a b}$, such that $\mathcal{I}^{m}=0$ for all $m \geqslant 3$. If $\operatorname{Hom}\left(\mathrm{H}_{i}(\mathbf{M}), \mathcal{I}^{2}\right)=0$ for all $i \geqslant 1$, then for all $n \geqslant 1$ there exists an exact and natural sequence

$$
\begin{gathered}
0 \longrightarrow \operatorname{Ext}_{\mathcal{C M}_{a b}}^{1}\left(\mathrm{H}_{n}(\mathbf{M}), \mathbf{A}\right) \longrightarrow \mathrm{H}^{n+1}(\mathbf{M}, \mathbf{A}) \\
\longrightarrow \operatorname{Hom}\left(\mathrm{H}_{n+1}(\mathbf{M}), \mathbf{A}\right) \longrightarrow 0
\end{gathered}
$$

Moreover, there exists an isomorphism $\boldsymbol{\Theta}$ between $\operatorname{Cext}(\mathbf{M}, \mathbf{A})$ and $\mathrm{H}^{2}(\mathbf{M}, \mathbf{A})$, [4].

Definition 2. The central extension e is called a quasi-commutator extension if $\theta_{\star}(e)$ is injective.

Note that the map $\theta_{\star}(e)$ is injective if and only if $\left(\pi_{1}, \pi_{2}\right)_{\star}: \mathrm{H}_{2}(\mathbf{T}) \rightarrow \mathrm{H}_{2}(\mathbf{M})$ has trivial image, that is, if and only if $\left(\pi_{1}, \pi_{2}\right)_{\star}$ is the zero map. Thus

$$
\mathbf{0} \longrightarrow \mathrm{H}_{2}(\mathbf{M}) \stackrel{\theta_{\star}(e)}{\longrightarrow}(N, R, \nu) \stackrel{\left(\chi_{1}, \chi_{2}\right)_{\star}}{\longrightarrow} \mathrm{H}_{1}(\mathbf{T}) \longrightarrow \mathrm{H}_{1}(\mathbf{M}) \longrightarrow \mathbf{0}
$$

is an exact sequence.

From $[\mathbf{1 1}], \theta_{\star}(e)$ can be factored as a surjective and an injective morphisms:

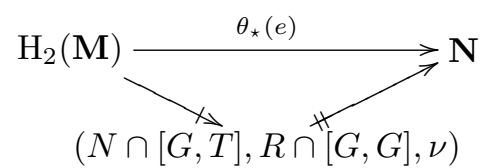

hence, $\theta_{\star}(e)$ is an injective morphism if and only if $\mathrm{H}_{2}(\mathbf{M}) \cong(N \cap[G, T], R \cap[G, G], \nu)$. In fact, if $\mathrm{H}_{2}(\mathbf{M})=\mathbf{0}$, then $e$ is a commutator extension.

Definition 3. The central extension $e$ is called a stem extension when $\theta_{\star}(e)$ is surjective.

Proposition 1. The following statements are equivalent:

i) e is a stem extension,

ii) $\mathbf{N} \rightarrow \mathrm{H}_{1}(\mathbf{T})$ is the trivial map,

iii) $\left(\pi_{1}, \pi_{2}\right)_{\star}: \mathrm{H}_{1}(\mathbf{T}) \rightarrow \mathrm{H}_{1}(\mathbf{M})$ is an isomorphism,

iv) $\mathbf{N} \subseteq[\mathbf{T}, \mathbf{T}]$

The proof is very easy by using the five-term homology sequence exactness in Theorem 1 and the cross lemma. 
Definition 4. The central extension e is called a stem cover if $\theta_{\star}(e)$ is an isomorphism.

It is trivial to prove the following result

Proposition 2. The following statements are equivalent:

1. e is a stem cover,

2. $\mathrm{H}_{2}(\mathbf{M}) \cong \mathbf{N}$,

3. $\mathbf{T}_{a b} \cong \mathbf{M}_{a b}$ and $\left(\pi_{1}, \pi_{2}\right)_{\star}: \mathrm{H}_{2}(\mathbf{T}) \rightarrow \mathrm{H}_{2}(\mathbf{M})$ is the zero map.

If we write Definitions 1,3 and 4 and Propositions 1, 2 and 3 for the crossed modules $(G, G, i d)$ and $(1, G, i)$, then we obtain definition and caracterization in [15] for theoretical group central extensions.

\section{Stem extensions and stem covers of perfect crossed mod- ules}

From now, let us consider $\mathbf{M}$ a perfect crossed module, that is $\mathbf{M}=[\mathbf{M}, \mathbf{M}]$; in this situation $\mathrm{H}^{2}(\mathbf{M}, \mathbf{N}) \cong \operatorname{Hom}\left(\mathrm{H}_{2}(\mathbf{M}), \mathbf{N}\right)$, from Theorem 4 .

Proposition 3. Let $\mathbf{N}$ be a trivial $\mathbf{M}$-module. Every central extension class of $\mathbf{N}$ by $\mathbf{M}$ is forward induced from a stem extension.

Proof:

We consider $\left[e: \mathbf{N} \# \stackrel{\left(\chi_{1}, \chi_{2}\right)}{\longrightarrow} \mathbf{T} \stackrel{\left(\pi_{1}, \pi_{2}\right)}{\longrightarrow} \longrightarrow \mathbf{M}\right]$,

$$
[e] \in \operatorname{Cext}(\mathbf{M}, \mathbf{N}) \stackrel{\Theta}{\cong} \mathrm{H}^{2}(\mathbf{M}, \mathbf{N}) \cong \operatorname{Hom}\left(\mathrm{H}_{2}(\mathbf{M}), \mathbf{N}\right) \text {. }
$$

It is also known the factorization of $\theta_{\star}(e)$

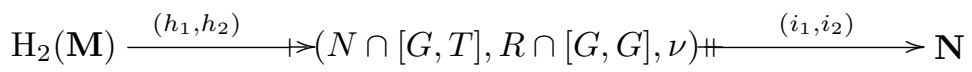

(see [11]), where $\mathbf{N} \cap[\mathbf{T}, \mathbf{T}]$ is also $\mathbf{M}$-trivial. Then, by Theorem 4 , there exists $\left[e_{1}\right] \in$ $\operatorname{Cext}(\mathbf{M}, \mathbf{N} \cap[\mathbf{T}, \mathbf{T}])$ such that $\theta_{\star}\left(e_{1}\right)=\left(h_{1}, h_{2}\right)$ (hence $e_{1}$ is a stem-extension), and we can consider the extension morphism $\left(\left(i_{1}, i_{2}\right),-, i d\right): e_{1} \rightarrow^{\left(i_{1}, i_{2}\right)} e_{1}$ and so, by $\theta_{\star}$ naturality, $\theta_{\star}(e)=\left(i_{1}, i_{2}\right)\left(h_{1}, h_{2}\right)=\left(i_{1}, i_{2}\right) \theta_{\star}\left(e_{1}\right)=\theta_{\star}\left(\left(i_{1}, i_{2}\right)_{\star}\left(e_{1}\right)\right)$. Thus, $\left(i_{1}, i_{2}\right)_{\star}\left(e_{1}\right) \equiv e$.

Proposition 4. Let $\mathbf{U}=(U, V, \omega)$ be a subcrossed module of $\mathrm{H}_{2}(\mathbf{M})$. Then there exists a stem extension $e$ with $\mathbf{U}=\operatorname{Ker} \theta_{\star}(e)$.

Proof:

Let $\mathbf{N}=\frac{\mathrm{H}_{2}(\mathbf{M})}{\mathbf{U}}$. By applying Theorem 4, we choose any central extension $e: \mathbf{N} \# \longrightarrow \mathbf{T} \longrightarrow \mathbf{M}$ with $\theta_{\star}([e])$ the canonical projection $\mathrm{H}_{2}(\mathbf{M}) \rightarrow \mathbf{N}$. Then $\operatorname{Ker} \theta_{\star}(e)=\mathbf{U}$ being $e$ a stem extension. 
It is known that a stem extension $e$ is a stem cover if and only if $\theta_{\star}(e)$ is also injective. If $\theta_{\star}(e)$ is an isomorphism, we can consider its inverse $\left(\varphi_{1}, \varphi_{2}\right): \mathbf{N} \rightarrow$ $\mathrm{H}_{2}(\mathbf{M})$. Theorem 4 allows us to consider the following commutative diagram

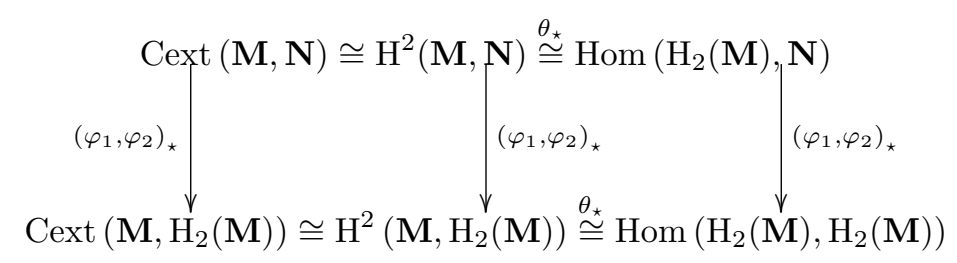

(see also [4]), and thus $\left(\varphi_{1}, \varphi_{2}\right)_{\star}\left(\theta_{\star}(e)\right)=\theta_{\star}\left(\left(\varphi_{1}, \varphi_{2}\right) e\right)=i d_{\mathrm{H}_{2}(\mathbf{M})}$. Hence, any stem cover $e$ is isomorphic to a stem cover

$$
e^{\prime}: \mathrm{H}_{2}(\mathbf{M}) \Perp \mathbf{T}^{\prime} \longrightarrow \mathbf{M}
$$

with $\theta_{\star}\left(e^{\prime}\right)=i d_{\mathrm{H}_{2}(\mathbf{M})}$. Since we have the commutative diagram

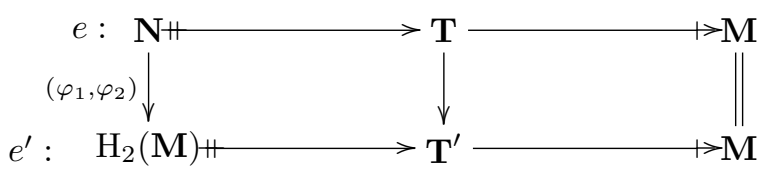

we can say that $e$ and $e^{\prime}$ belong to the same isomophism class [16].

From this and from Definition 4 we conclude the following

Proposition 5. For a perfect crossed module $(M, P, \mu)$, there exists only one isomorphism class of stem covers of $\mathbf{M}$.

Proposition 6. Every stem extension of the perfect crossed module $\mathbf{M}$ is epimorphic image of some stem cover.

Proof:

Let $e: \mathbf{N} \Perp\left(\chi_{1}, \chi_{2}\right) \longrightarrow \mathbf{T} \stackrel{\left(\pi_{1}, \pi_{2}\right)}{\longrightarrow} \longrightarrow \mathbf{M}$ be a stem extension; from Theorem 2 , we have the morphism

$$
\theta^{\star}: \operatorname{Hom}_{\mathbf{M}}(\mathbf{N}, \mathbf{N}) \rightarrow \operatorname{Cext}(\mathbf{M}, \mathbf{N}),
$$

given by $\theta^{\star}\left(h_{1}, h_{2}\right)=\left[{ }^{\left(h_{1}, h_{2}\right)} e\right]$, (also see [16]); thus $e$ can be a stem extension characterized by $\theta^{\star}\left(i d_{\mathbf{N}}\right)=[e] \in \operatorname{Cext}(\mathbf{M}, \mathbf{N})$, that is, an element $\xi \in \mathrm{H}^{2}(\mathbf{M}, \mathbf{N}) \cong$ $\operatorname{Cext}(\mathbf{M}, \mathbf{N})$. Then we have an epimorphism

$$
\theta_{\star}(\Theta[e])=\theta_{\star}(\xi)=\left(\varphi_{1}, \varphi_{2}\right)=\theta_{\star}(\xi): \mathrm{H}_{2}(\mathbf{M}) \rightarrow \mathbf{N} .
$$

We have, from Theorem 4 , the commutative square

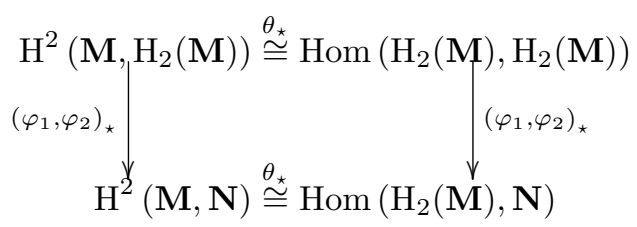


Let $\xi^{\prime} \in \mathrm{H}^{2}\left(\mathbf{M}, \mathrm{H}_{2}(\mathbf{M})\right)$ with $\theta_{\star}\left(\xi^{\prime}\right)=i d_{\mathrm{H}_{2}(\mathbf{M})}$; then $\theta_{\star}\left(\xi-\left(\varphi_{1}, \varphi_{2}\right)_{\star} \xi^{\prime}\right)=\theta_{\star}(\xi)-$ $\left(\varphi_{1}, \varphi_{2}\right) \theta_{\star}\left(\xi^{\prime}\right)=0$. Thus $\xi=\left(\varphi_{1}, \varphi_{2}\right)_{\star} \xi^{\prime}$.

Hence, there exists $\left[e^{\prime}\right] \in \operatorname{Cext}\left(\mathbf{M}, \mathrm{H}_{2}(\mathbf{N})\right)$ with $\Theta\left[e^{\prime}\right]=\xi^{\prime}$. So $\left(\varphi_{1}, \varphi_{2}\right)_{\star}\left[e^{\prime}\right]=[e]$, where $\left(\varphi_{1}, \varphi_{2}\right)_{\star}$ is an ephimorphism.

Proposition 7. Let $e^{\prime}: \mathrm{H}_{2}\left(\mathbf{M}^{\prime}\right)+\longrightarrow \mathbf{T}^{\prime} \longrightarrow \mathbf{M}^{\prime}$ be a stem cover and $e: \mathbf{N} \stackrel{\left(\chi_{1}, \chi_{2}\right)}{\longrightarrow} \mathbf{T} \stackrel{\left(\pi_{1}, \pi_{2}\right)}{\longrightarrow} \longrightarrow \mathbf{M}$ be a stem extension with $\mathbf{M}^{\prime}$ and $\mathbf{M}$ perfect crossed modules. Then every homomorphism $\left(f_{1}, f_{2}\right): \mathbf{M}^{\prime} \rightarrow \mathbf{M}$ can be lifted to a map $\left(f_{1}^{\prime}, f_{2}^{\prime}\right): \mathbf{T}^{\prime} \rightarrow \mathbf{T}$.

Proof:

If $\left(f_{1}, f_{2}\right)$ is given, Theorem 4 allows us to construct the following commutative diagram

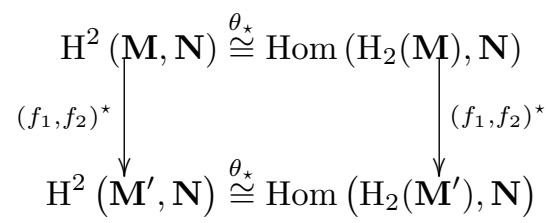

As we have been doing, we identify $\xi \in \mathrm{H}^{2}(\mathbf{M}, \mathbf{N})$ with $[e]$ via the isomorphism $($ see $[4]) \Theta: \operatorname{Cext}(\mathbf{M}, \mathbf{N}) \rightarrow H^{2}(\mathbf{M}, \mathbf{N})$, and we consider the morphism $\left(\varphi_{1}, \varphi_{2}\right)=$ $\left(f_{1}, f_{2}\right)^{\star}\left(\theta_{\star}(\xi)\right) \in \operatorname{Hom}\left(\mathrm{H}_{2}\left(\mathbf{M}^{\prime}\right), \mathbf{N}\right)$ to construct the diagram

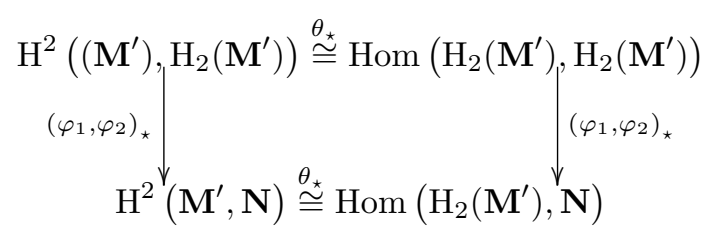

again from Theorem 4. Since $\boldsymbol{\Theta}\left[e^{\prime}\right]=\xi^{\prime}$ is a stem cover, we can suppose that $\theta_{\star}\left(\xi^{\prime}\right)=i d_{\mathrm{H}_{2}\left(\mathbf{M}^{\prime}\right)}$. Thus $\left(\varphi_{1}, \varphi_{2}\right)_{\star}\left(\theta_{\star}\left(\xi^{\prime}\right)\right)=\left(\varphi_{1}, \varphi_{2}\right)$ from (2) commutativity. So $\left(\varphi_{1}, \varphi_{2}\right)_{\star}\left(\xi^{\prime}\right)=\left(f_{1}, f_{2}\right)^{\star}(\xi)$. The conclusion can be obtained by applying Theorem 2.1.1 from [16], and seeing $[4,9]$.

Proposition 8. Let $e: \mathbf{N} \# \longrightarrow \mathbf{T} \longrightarrow \longrightarrow \mathbf{M}$ and $e^{\prime}: \mathbf{N}^{\prime} \# \longrightarrow \mathbf{T}^{\prime} \longrightarrow \mathbf{M}^{\prime}$ be two central extensions with $\mathbf{M}$ and $\mathbf{M}^{\prime}$ perfect crossed modules. Also consider $\left(f_{1}, f_{2}\right)$ : $\mathbf{N} \rightarrow \mathbf{N}^{\prime}$ and $\left(h_{1}, h_{2}\right): \mathbf{M} \rightarrow \mathbf{M}^{\prime}$ two crossed module morphisms. There exists a unique morphism $\left(\omega_{1}, \omega_{2}\right): \mathbf{T} \rightarrow \mathbf{T}^{\prime}$ such that $\left(\left(f_{1}, f_{2}\right),\left(\omega_{1}, \omega_{2}\right),\left(h_{1}, h_{2}\right)\right): e \rightarrow e^{\prime}$ is a morphism of extensions if and only if

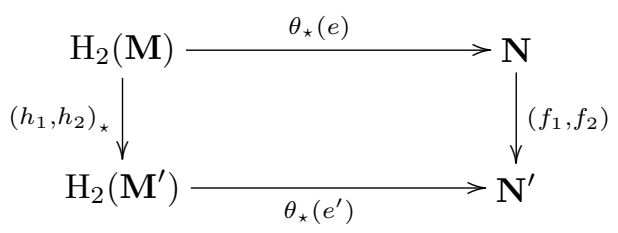

is a commutative square. 


\section{Proof:}

If $\left(\left(f_{1}, f_{2}\right),\left(\omega_{1}, \omega_{2}\right),\left(h_{1}, h_{2}\right)\right)$ exists, then the square (3) is commutative, from Theorem 1. Conversely, Theorem 4 gives up the diagram

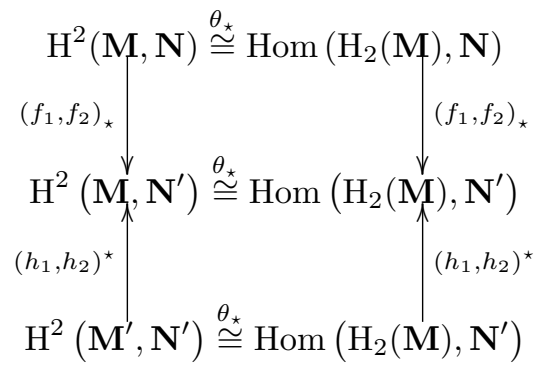

Let $\xi \in \mathrm{H}^{2}(\mathbf{M}, \mathbf{N})$ and $\xi^{\prime} \in \mathrm{H}^{2}\left(\mathbf{M}^{\prime}, \mathbf{N}^{\prime}\right)$ be the corresponding element to $[e]$ and $\left[e^{\prime}\right]$, respectively, by the isomorphism $\boldsymbol{\Theta}$. From the above diagram and since $\left(f_{1}, f_{2}\right) \theta_{\star}(e)=\theta_{\star}\left(e^{\prime}\right)\left(h_{1}, h_{2}\right)_{\star}$, we have $\left(f_{1}, f_{2}\right)_{\star}(\xi)=\left(h_{1}, h_{2}\right)^{\star}\left(\xi^{\prime}\right) \in \mathrm{H}^{2}\left(\mathbf{M}, \mathbf{N}^{\prime}\right)$.

For getting the existence and uniqueness of $\left(\omega_{1}, \omega_{2}\right)$ see Theorem 2.1.1 from [16].

Proposition 9. Let $e: \mathbf{N} \mathbb{(}^{\left(\chi_{1}, \chi_{2}\right)} \longrightarrow \mathbf{T} \stackrel{\left(\pi_{1}, \pi_{2}\right)}{\longrightarrow} \longrightarrow \mathbf{M}$ be a central extension and let $\left(f_{1}, f_{2}\right): \mathbf{M}^{\prime} \rightarrow \mathbf{M}$ be a morphism of crossed modules where $\mathbf{M}^{\prime}$ and $\mathbf{M}$ are perfect crossed modules. Then there exists a morphism of crossed modules $\left(\varphi_{1}, \varphi_{2}\right)$ : $\mathbf{M}^{\prime} \rightarrow \mathbf{T}$ verifying $\left(\pi_{1}, \pi_{2}\right)\left(\varphi_{1}, \varphi_{2}\right)=\left(f_{1}, f_{2}\right)$ if and only if $\left(f_{1}, f_{2}\right)_{\star}\left(\mathrm{H}_{2}\left(\mathbf{M}^{\prime}\right)\right) \subseteq$ $\left(\pi_{1}, \pi_{2}\right)_{\star}\left(\mathrm{H}_{2}(\mathbf{T})\right)$. If $\left(\varphi_{1}, \varphi_{2}\right)$ exists, it is uniquely determined.

\section{Proof:}

If $\left(\varphi_{1}, \varphi_{2}\right)$ exists, since $\mathrm{H}_{2}(-)$ preserves de composition,

$$
\left(f_{1}, f_{2}\right)_{\star}\left(\mathrm{H}_{2}\left(\mathbf{M}^{\prime}\right)\right)=\left(\pi_{1}, \pi_{2}\right)_{\star}\left(\varphi_{1}, \varphi_{2}\right)_{\star}\left(\mathrm{H}_{2}\left(\mathbf{M}^{\prime}\right)\right) \subseteq\left(\pi_{1}, \pi_{2}\right)_{\star}\left(\mathrm{H}_{2}(\mathbf{T})\right) .
$$

Conversely, by considering the exact sequence

$$
\operatorname{Ker}\left(f_{1}, f_{2}\right) \# \longrightarrow \mathbf{M}^{\prime} \stackrel{\left(f_{1}, f_{2}\right)}{\longrightarrow} \gg \operatorname{Im}\left(f_{1}, f_{2}\right),
$$

we get the following one

$$
e^{\prime}: \frac{\operatorname{Ker}\left(f_{1}, f_{2}\right)}{\left[\operatorname{Ker}\left(f_{1}, f_{2}\right), \mathbf{M}^{\prime}\right]} \Psi \frac{\mathbf{M}^{\prime}}{\left[\operatorname{Ker}\left(f_{1}, f_{2}\right), \mathbf{M}^{\prime}\right]} \longrightarrow \frac{\left(f_{1}^{\prime}, f_{2}^{\prime}\right)}{1 \rightarrow \operatorname{Im}}\left(f_{1}, f_{2}\right),
$$

where $\left(f_{1}^{\prime}, f_{2}^{\prime}\right)$ is induced by $\left(f_{1}, f_{2}\right)$. Let

$$
\mathbf{N}^{\prime}=\frac{\operatorname{Ker}\left(f_{1}, f_{2}\right)}{\left[\operatorname{Ker}\left(f_{1}, f_{2}\right), \mathbf{M}^{\prime}\right]} \text { and }\left(T^{\prime}, G^{\prime}, \partial^{\prime}\right)=\frac{\mathbf{M}^{\prime}}{\left[\operatorname{Ker}\left(f_{1}, f_{2}\right), \mathbf{M}^{\prime}\right]} ;
$$

then $\left(f_{1}^{\prime}, f_{2}^{\prime}\right)_{\star}\left(\mathrm{H}_{2}(\mathbf{T})\right)=\left(f_{1}, f_{2}\right)_{\star}\left(\mathrm{H}_{2}\left(\mathbf{M}^{\prime}\right)\right)$.

Since $\mathbf{M}^{\prime}$ is perfect and $\left(f_{1}^{\prime}, f_{2}^{\prime}\right)_{\star}\left(\mathrm{H}_{2}(\mathbf{T})\right) \subseteq\left(\pi_{1}, \pi_{2}\right)_{\star}\left(\mathrm{H}_{2}(\mathbf{T})\right)$, there exists an injective map $\operatorname{Im}\left(f_{1}^{\prime}, f_{2}^{\prime}\right)_{\star} \rightarrow \operatorname{Im}\left(\pi_{1}, \pi_{2}\right)_{\star}$ that induces a morphism $\left(h_{1}, h_{2}\right): \mathbf{N}^{\prime} \rightarrow$ $\mathbf{N}$. From Proposition 8, the existence of $\left(\omega_{1}, \omega_{2}\right): \mathbf{T}^{\prime} \rightarrow \mathbf{T}$ is followed; this morphism 
yields a morphism $\left(\left(h_{1}, h_{2}\right),\left(\omega_{1}, \omega_{2}\right), i n c\right): e^{\prime} \rightarrow e$. Now, the morphism $\left(\varphi_{1}, \varphi_{2}\right)$ can be obtained as the composition $\mathbf{M}^{\prime} \longrightarrow \mathbf{T}^{\prime} \stackrel{\left(\omega_{1}, \omega_{2}\right)}{\longrightarrow} \mathbf{T}$.

Theorem 5. The isomorphism classes of stem extensions of the perfect crossed module $\mathbf{M}$ are in bijective correspondence with the subcrossed modules of $\mathrm{H}_{2}(\mathbf{M})$. Moreover, if $\mathbf{U}$ and $\mathbf{U}^{\prime}$ are two subcrossed modules of $\mathrm{H}_{2}(\mathbf{M})$, then $\mathbf{U} \subseteq \mathbf{U}^{\prime}$ if and only if there is a map from the stem extension corresponding to $\mathbf{U}$ to the corresponding one to $\mathbf{U}^{\prime}$.

\section{Proof:}

Since there is only one isomorphism class of stem cover of $\mathbf{M}$ from Proposition 5, if we consider a stem extension $e: \mathbf{N} \# \longrightarrow \mathbf{M}$, then we get $\mathbf{U}=\operatorname{Ker}\left(\theta_{\star}(e): \mathrm{H}_{2}(\mathbf{M}) \rightarrow \mathbf{N}\right)$, a subcrossed module of $\mathrm{H}_{2}(\mathbf{M})$, and another stem extension isomorphic to $e$ will yield the same subcrossed module.

Conversely, let $\mathbf{U} \subseteq \mathrm{H}_{2}(\mathbf{M})$ be given, and $\mathbf{N}=\frac{\mathrm{H}_{2}(\mathbf{M})}{\mathbf{U}}$. We can consider the canonical projection $\left(q_{1}, q_{2}\right): \mathrm{H}_{2}(\mathbf{M}) \rightarrow \mathbf{N}$ and so, from Theorem 4, there exists a unique $\xi \in \mathrm{H}^{2}(\mathbf{M}, \mathbf{N})$ such that $\theta_{\star}(\xi)=\left(q_{1}, q_{2}\right)$, being $\xi$ the corresponding to $[e] \in \operatorname{Cext}(\mathbf{M}, \mathbf{N})$ by the isomorphism $\boldsymbol{\Theta}$. Since $\theta_{\star}(e)=\left(q_{1}, q_{2}\right)$, is an epimorphism, $e$ is a stem extension such that $(U, V, \omega)=\operatorname{Ker}\left(\theta_{\star}(e): \mathrm{H}_{2}(\mathbf{M}) \rightarrow \mathbf{N}\right)$.

If $\left[e^{\prime}\right] \in \operatorname{Cext}\left(\mathbf{M}, \mathbf{N}^{\prime}\right)$ is another stem extension which verifies $\mathbf{U}=\operatorname{Ker} \theta_{\star}\left(e^{\prime}\right)$, we can construct the following commutative diagram

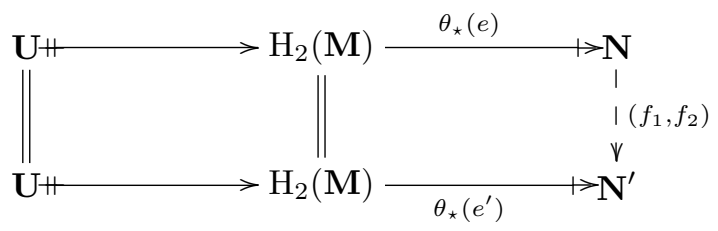

Obviously, $\left(f_{1}, f_{2}\right)$ is an isomorphism, so $e$ and $e^{\prime}$ are in the conditions of Proposition 8 and we have a morphism of extensions $\left(\left(f_{1}, f_{2}\right),\left(\omega_{1}, \omega_{2}\right), i d_{\mathbf{M}}\right): e \rightarrow e^{\prime}$. Hence $e$ and $e^{\prime}$ belong to the same isomorphism class

Now we consider an extension morphism $\left(\left(f_{1}, f_{2}\right),\left(\omega_{1}, \omega_{2}\right), i d_{\mathbf{M}}\right): e \rightarrow e^{\prime}$, where $e$ and $e^{\prime}$ are stem extensions of $\mathbf{M}$ with $\mathbf{U}=\operatorname{Ker} \theta_{\star}(e)$ and $\mathbf{U}^{\prime}=\operatorname{Ker} \theta_{\star}\left(e^{\prime}\right)$; then we construct the commutative square

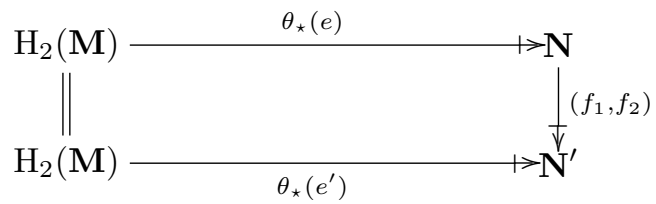

Thus, there exists $\left(h_{1}, h_{2}\right): \mathbf{U} \# \longrightarrow \mathbf{U}^{\prime}$.

Conversely, let $\mathbf{U} \subseteq \mathbf{U}^{\prime} \subseteq \mathrm{H}_{2}(\mathbf{M})$ be given, and denote $\mathbf{N}=\mathrm{H}_{2}(\mathbf{M}) / \mathbf{U}$ and $\mathbf{N}^{\prime}=\mathrm{H}_{2}(\mathbf{U}) / \mathbf{U}^{\prime}$. Since from Proposition 4 and the previous results obtained in this proof we can say that every stem extension is isomorphic to a stem extension $e$ with $\theta_{\star}(e)$ the canonical projection, we get the following commutative diagram of 
canonical projections

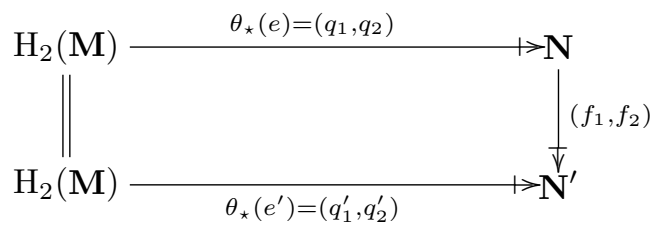

We now can apply Proposition 8 which yields the existence of

$$
\left(\left(f_{1}, f_{2}\right),\left(\omega_{1}, \omega_{2}\right), i d_{\mathbf{M}}\right): e \rightarrow e^{\prime},
$$

where $\left(\omega_{1}, \omega_{2}\right)$ is an epimorphism since $\left(f_{1}, f_{2}\right)$ also is.

Proposition 10. If $\mathbf{M}$ is perfect and $e: \mathbf{N} \Vdash \longrightarrow \mathbf{M}$, is a

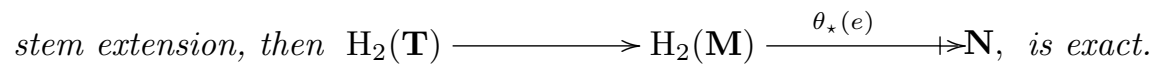

The proof is easy by seeing Theorem 1 .

Corollary 1. Let $\mathbf{M}$ be perfect and let $e: \mathbf{N H} \longrightarrow \mathbf{T} \longrightarrow \mathbf{M}$, be a central extension. If $\mathrm{H}_{1}(\mathbf{T})$ and $\mathrm{H}_{2}(\mathbf{T})$ are trivial crossed modules, then $e$ is a stem cover of $\mathbf{M}$.

Corollary 2. Let $e: \mathbf{N}+\longrightarrow \mathbf{T} \longrightarrow \mathbf{M}$, be an universal central extension of crossed modules, then is a stem cover.

\section{Proof:}

By theorem 2.60 in $[\mathbf{1 3}], \mathbf{T}$ is a perfect crossed module, then $\mathrm{H}_{1}(\mathbf{T})=0$. More-

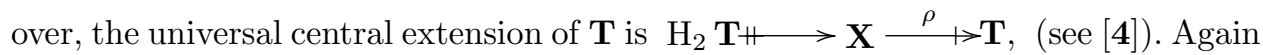
by Theorem 2.60 in $[\mathbf{1 3}]$ this sequence is split, then $\mathrm{H}_{2}(\mathbf{T})=0$. Thus Corollary 1 says that $e$ is a stem cover.

\section{Examples:}

1. Let $I$ be a two-sided ideal of a ring $R$ and let $\operatorname{GL}(R, I)$ be the kernel of $\mathrm{GL}(R) \rightarrow \mathrm{GL}(R / I)$ and $\mathrm{E}(I)=\mathrm{E}(R) \cap \mathrm{GL}(R, I)$, where $\mathrm{E}(R)$ is the subgroup of the infinite general linear group $\mathrm{GL}(R)$ generated by the elementary matrices. Then $(\mathrm{E}(I), \mathrm{E}(R), i)$, is a perfect crossed module and its universal central extension is

$$
1 \rightarrow\left(\mathrm{K}_{2}(R, I), \mathrm{K}_{2}(R), \delta\right) \rightarrow(\mathrm{St}(R, I), \operatorname{St}(R), \delta) \rightarrow(\mathrm{E}(I), \mathrm{E}(R), i) \rightarrow 1
$$

where $\operatorname{St}(R)$ and $\operatorname{St}(R, I)$ are the Steinberg and relative Steinberg groups respectively $[\mathbf{6}, \mathbf{1 2}]$. This extension is a stem cover, by Corollary 2, and $\mathrm{H}_{2}(\operatorname{St}(R, I), \mathrm{St}(R), \delta)=0$. (Further information about Steinberg groups can be seen in $[8])$.

2. Let $A$ be a $G$-module, where $G$ is a perfect group. We know that

$$
[(A, G, 0),(A, G, 0)]=(A \circ I G, G, 0)
$$


where $I G$ is the augmentation ideal of $\mathbb{Z} G \rightarrow \mathbb{Z},[\mathbf{1 6}]$. Let $B$ be the subgroup of $A$ generated by $\left\{\left({ }^{g} a\right)-2 a \mid g \in G, a \in A\right\}$. Then $(A / B, G, 0)$ is a perfect crossed module and its universal central extension is

$$
1 \rightarrow\left(\mathrm{H}_{1}(G, A / B), \mathrm{H}_{2}(G), 0\right) \rightarrow((A / B) \otimes G, G \otimes G, 0) \rightarrow(A / B, G, 0) \rightarrow 1
$$

where $\otimes$ denotes the non abelian tensor product of Brown and Loday (see [3, 8]). By Corollary 2, this universal central extension is a stem cover. Moreover, $\mathrm{H}_{2}((A / B) \otimes G, G \otimes G, 0)=0$. Since

$$
\mathrm{H}_{2}((A / B) \otimes G, G \otimes G, 0)=\left(\mathrm{H}_{1}(G \otimes G,(A / B) \otimes G), \mathrm{H}_{2}(G \otimes G), 0\right)
$$

then $\mathrm{H}_{1}(G \otimes G,(A / B) \otimes G)=0$ and $\mathrm{H}_{2}(G \otimes G)=0$.

\section{References}

[1] H.-J. BAUES. Non-abelian extensions and homotopies. $K$-Theory 10, No. 2 (1996), 107-133.

[2] F. R. Beyl and J. TAPPE, "Group Extensions, Representations, and the Schur Multiplicator". Lecture Notes in Math. 958, Springer-Verlag, Berlin-New York, 1982.

[3] R. Brown and J.-L. Loday, Van Kanpen Theorems for diagram of spaces. Topology 26 (1987), 311-335.

[4] P. Carrasco, A. M. Cegarra and A. R. Grandjeán, (Co)Homology of crossed modules in Category Theory Meeting (CT 99). Coimbra, Portugal (1999).

[5] G. J. Ellis, Homology of 2-types, J. London Math. Soc. (2) 46, No. 1 (1992), 1-27.

[6] N.D. Gilbert, The low dimensional homology of crossed modules, Homology, Homotopy and Applications 2 (2000), 41-50.

[7] A. R.-GrandjeÁn and M. Ladra, $\mathrm{H}_{2}(T, G, \partial)$ and central extensions for crossed modules, Procc. Edinburg Math. Soc. 42 (1999), 169-177.

[8] A. R.-GrandjeÁn and M. P. LóPez, $\mathrm{H}_{2}(T, G, \partial)$ and $q$-perfect crossed modules. To appear in Applied Categorical Structures.

[9] P. J. Hilton and U. Stammbach, A Course in Homological Algebra (Graduate Texts in Mathematics 4, Springer-Verlag, 1971).

[10] J. Huebschmann, Crossed $n$-fold extensions of groups and cohomology, Comment. Math. Helvetici 55 (1980), 302-314.

[11] M. Ladra and A. R.-Grandjeán, Crossed modules and homology, $J$. P. App. Algebra 95 (1994), 41-55.

[12] J.-L. LodAy, Cohomologie et groupe de Steinberg relatifs, J. Algebra 54 (1978), 178-202.

[13] K. J. NorRIE, Actions and automorphims of crossed modules, Bull. Soc. Math. France 118 (1990), 129-146. 
[14] J.G. Ratcliffe, Crossed extensions, Trans. Am. Math. Soc. 257, n. 1 (1980), 73-89.

[15] U. Stammbach, "Homology in group theory". Lecture Notes in Math. 359, Springer-Verlag, Berlin-New York. 1973.

[16] A. M. Vieites, Extensiones Abelianas, Cruzadas y 2-Extensiones Cruzadas de Módulos Cruzados (Ph. D. Thesis, Universidad de Vigo, 1999).

[17] A. M. Vieites and J. M. Casas, Derivations of Crossed Modules, Extracta Mathematicae 14, n.1 (1999) , 63-67.

[18] A. M. Vieites, J. M. Casas and M. Ladra, n-Crossed extensions of Crossed Modules (Preprint, 1999).

This article may be accessed via WWW at http://www.rmi.acnet.ge/hha/ or by anonymous ftp at

ftp://ftp.rmi.acnet.ge/pub/hha/volumes/2002/n1a3/v4n1a3.(dvi,ps,pdf)

A. M. Vieites avieites@dma.uvigo.es

Dpto. Matemática Aplicada.

Universidad de Vigo.

36280 Vigo (Pontevedra).

Spain

J. M. Casas jmcasas@uvigo.es

Dpto. Matemática Aplicada.

Universidad de Vigo.

36280 Vigo (Pontevedra).

Spain 